\title{
ON THE CONVERGENCE OF A SEQUENCE OF PERRON INTEGRALS ${ }^{1}$
}

\author{
MANOUG N. MANOUGIAN
}

Introduction. This paper is concerned with the convergence of a sequence of Perron integrals. Oscar Perron [4] considered the case of a sequence of uniformly convergent Perron integrable functions, and Bauer [1] extended Perron's work to functions defined in a space of $n$-dimensions. McShane [3] relaxed the condition of a uniformly convergent sequence of Perron integrable functions and stated necessary conditions for the limit of a sequence of Perron integrals to be the integral of the limit function. The following theorem is a generalization of the above. Throughout this paper integration is in the Perron sense. The Lebesgue integral is denoted by $(\mathscr{L}) \int$.

Theorem. H1. $\left\{f_{n}(x)\right\}$ is a sequence of Perron integrable functions whose domain is $I=\{x \mid a \leqq x \leqq b\}$.

H2. $f_{n}(x) \geqq g(x)$ for each $n$, a.e. (almost everywhere) on $I$, where $g(x)$ is Perron integrable on $I$.

H3. $\lim _{n} f_{n}(x)=f(x)$ a.e. on $I$.

Under hypotheses $\mathrm{H} 1-\mathrm{H} 3, f(x)$ is Perron integrable on $I$, and $\lim _{n} \int_{a}^{x} f_{n}(t) d t=\int_{a}^{x} f(t) d t$, if and only if the sequence of integrals $\left\{\int_{a}^{x}\left[f_{n}(t)-g(t)\right] d t\right\}$ is EAC (equi-absolutely continuous) on $I$.

Preliminary theorems. The following theorems are used in the proof of the theorem above. The reader is referred to Kamke [2] or McShane [3] for a proof of Theorem 1, Theorem 2, and Theorem 3. Vitali [5] gave a proof for Theorem 4 .

THEOREM 1. If each of $f_{1}(x)$ and $f_{2}(x)$ is a Perron integrable function on $I$, and $k_{1}$ and $k_{2}$ are numbers, then $\left[k_{1} f_{1}(x)+k_{2} f_{2}(x)\right]$ is Perron integrable on $I$, and

$$
\int_{a}^{x}\left[k_{1} f_{1}(t)+k_{2} f_{2}(t)\right] d t=k_{1} \int_{a}^{x} f_{1}(t) d t+k_{2} \int_{a}^{x} f_{2}(t) d t .
$$

Theorem 2. If $f(x)$ is Lebesgue integrable on $I$, then $f(x)$ is Perron integrable on I and

Presented to the Society, April 12, 1968; received by the editors September 20, 1968.

1 This is a part of the author's doctoral dissertation, written at the University of Texas at Austin under the direction of Professor H. J. Ettlinger. 


$$
\int_{a}^{x} f(t) d t=(\mathfrak{L}) \int_{a}^{x} f(t) d t .
$$

Theorem 3. If $f(x)$ is Perron integrable on $I$, and $f(x) \geqq 0$ a.e. on $I$, then $f(x)$ is Lebesgue integrable on $I$.

THEOREM 4. If $\left\{f_{n}(x)\right\}$ is a sequence of Lebesgue integrable functions for $x$ on $I, \lim _{n} f_{n}(x)=f(x)$ a.e. on $I$, and $f_{n}(x) \geqq 0$ a.e. on $I$, then $f(x)$ is Lebesgue integrable and $\lim _{n}(\mathscr{L}) \int_{a}^{x} f_{n}(t) d t=(\mathscr{L}) \int_{a}^{x} f(t) d t$ if and only if the sequence $\left\{(\&) \int_{a}^{x} f_{n}(t) d t\right\}$ is $E A C$ on $I$.

Proof of Theorem. (i) Proof of the Sufficiency. Since $f_{n}(x) \geqq g(x)$ a.e. on $I$, then by Theorem 1, Theorem 2, and Theorem 3 we have for $x$ on $I$

$$
\int_{a}^{x}\left[f_{n}(t)-g(t)\right] d t=(\mathscr{L}) \int_{a}^{x}\left[f_{n}(t)-g(t)\right] d t
$$

and so the sequence $\left\{(\mathscr{L}) \int_{a}^{x}\left[f_{n}(t)-g(t)\right] d t\right\}$ is EAC on $I$. Then by Theorem 4,

$$
\lim _{n}(\mathfrak{L}) \int_{a}^{x}\left[f_{n}(t)-g(t)\right] d t=(\mathfrak{L}) \int_{a}^{x}[f(t)-g(t)] d t
$$

and Theorem 2 yields

$$
\lim _{n} \int_{a}^{x}\left[f_{n}(t)-g(t)\right] d t=\int_{a}^{x}[f(t)-g(t)] d t \quad \text { for } x \text { on } I .
$$

Now, since $g(x)$ is a Perron integrable function on $I$, then $f(x)$ is Perron integrable on $I$, and

$$
\lim _{n} \int_{a}^{x} f_{n}(t) d t=\int_{a}^{x} f(t) d t
$$

(ii) Proof of the Necessity. Under hypothesis,

$$
\lim _{n} \int_{a}^{x} f_{n}(t) d t=\int_{a}^{x} f(t) d t
$$

Then by Theorem 1 ,

$$
\lim _{n} \int_{a}^{x}\left[f_{n}(t)-g(t)\right] d t=\int_{a}^{x}[f(t)-g(t)] d t
$$

and by Theorem 3, 


$$
\lim \int_{a}^{x}\left[f_{n}(t)-g(t)\right] d t=\lim _{n}(\mathfrak{L}) \int_{a}^{x}\left[f_{n}(t)-g(t)\right] d t
$$

or,

$$
\lim _{n}(\mathfrak{L}) \int_{a}^{x}\left[f_{n}(t)-g(t)\right] d t=(\mathfrak{L}) \int_{a}^{x}[f(t)-g(t)] d t .
$$

Hence by Theorem 4 , the sequence $\left\{(\mathscr{L}) \int_{a}^{x}\left[f_{n}(t)-g(t)\right] d t\right\}$ is EAC on $I$, and Theorem 2 , yields the required result.

\section{REFERENCES}

1. H. Bauer, Der Perronsche Integralbegriff und seine Beziehung zum Lebesgueschen, Monatsh. Math. 26 (1915), 153-198.

2. E. Kamke, Das Lebesgue-Stieltjes-Integral, Teubner, Leipzig Verlagsgesellschaft, 1956.

3. E. J. McShane, Integration, Princeton Univ. Press, Princeton, N. J., 1944.

4. O. Perron, Über den Integralbegriff, Sitzungsberichte der Heidelberger Akademie der Wissenschaften, Ablielung A. Abhandlung 16 (1914). 155.

5. G. Vitali, Sull integrazione per serie, Rend. Circ. Mat. Palermo 23 (1907), 137-

UNIVERSITY OF SOUTH FLORIDA 